\title{
Order matters: sleep spindles contribute to memory consolidation only when followed by rapid-eye-movement sleep
}

\begin{abstract}
Authors
Mélanie Strauss [1,2,3], Lucie Griffon [1], Pascal Van Beers [1, 4], Maxime Elbaz [1], Jason Bouziotis [5], Fabien Sauvet [1, 4], Mounir Chenaoui [1, 4], Damien Léger [1] and Philippe Peigneux [2]
\end{abstract}

[1] Université de Paris, APHP, Hôtel-Dieu de Paris, Centre du Sommeil et de la Vigilance, EA 7330 VIFASOM Sommeil-Vigilance-Fatigue et Santé Publique, 75004 Paris, France.

[2] Neuropsychology and Functional Imaging Research Group (UR2NF) at Center for Research in Cognition and Neurosciences (CRCN) and ULB Neuroscience Institute (UNI), Université Libre de Bruxelles, 1050 Brussels, Belgium.

[3] Cliniques Universitaires de Bruxelles, Hôpital Erasme, Services de Neurologie, Psychiatrie et laboratoire du sommeil, Université Libre de Bruxelles, 1070 Brussels, Belgium.

[4] Unité Fatigue et vigilance, Institut de recherche biomédicale des armées, 91220 Brétigny sur Orge, France

[5] Cliniques Universitaires de Bruxelles, Hôpital Erasme, Service de la Recherche Biomédicale, Université Libre de Bruxelles, 1070 Brussels, Belgium.

(C) The Author(s) 2022. Published by Oxford University Press on behalf of Sleep Research Society. All rights reserved. For permissions, please e-mail: journals.permissions@oup.com 


\section{Corresponding author}

\section{Mélanie Strauss}

Services de Neurologie, psychiatrie et laboratoire du sommeil,

Cliniques Universitaires de Bruxelles, Hôpital Erasme,

Route de Lennik, 808

1070 Brussels,

Belgium.

melanie.strauss@erasme.ulb.ac.be 


\section{Abstract}

Sleep is known to benefit memory consolidation, but little is known about the contribution of sleep stages within the sleep cycle. The sequential hypothesis proposes that memories are first replayed during non-rapid-eye-movement (NREM or N) sleep and then integrated into existing networks during rapid-eye-movement (REM or R) sleep, two successive critical steps for memory consolidation. However, it lacks experimental evidence as $\mathrm{N}$ always precedes $\mathrm{R}$ sleep in physiological conditions. We tested this sequential hypothesis in patients with central hypersomnolence disorder, including patients with narcolepsy who present the unique, anti-physiological peculiarity of frequently falling asleep in $\mathrm{R}$ sleep before entering $\mathrm{N}$ sleep. Patients performed a visual perceptual learning task before and after daytime naps stopped after one sleep cycle, starting in $\mathrm{N}$ or $\mathrm{R}$ sleep and followed by the other stage (i.e. N-R vs. R-N sleep sequence). We compared over-nap changes in performance, reflecting memory consolidation, depending on the sleep sequence during the nap. Thirty-six patients who slept for a total of 67 naps were included in the analysis. Results show that sleep spindles are associated with memory consolidation only when $\mathrm{N}$ is followed by $\mathrm{R}$ sleep, that is in physiologically ordered N-R naps, thus providing support to the sequential hypothesis in humans. In addition, we found a negative effect of rapid-eye-movements in $\mathrm{R}$ sleep on perceptual consolidation, highlighting the complex role of sleep stages in the balance to remember and to forget.

\section{Keywords}

Sleep, sequential hypothesis, perceptual learning, memory consolidation, forgetting, sleep spindles, rapid-eye-movements, sleep cycles, narcolepsy 


\section{Statement of Significance}

Although increasing evidence shows that sleep plays an important role for memory consolidation processes, we do not know whether the systematically ordered succession of the different sleep stages within the sleep cycle matters for the processing of recent memories. Taking advantage of a rare disease, narcolepsy, we demonstrate that sleep spindles, an important marker of memory consolidation, are associated with improved over-nap performances only when the physiological sequence of sleep stages is respected, and not when the order of sleep stages is reversed. Those results reveal the importance of the physiological sleep sequence for memory consolidation, with sleep stages playing complementary and sequential roles. They thus provide experimental support to the sequential hypothesis of memory consolidation in humans. 


\section{Introduction}

Both non-rapid-eye-movements $(\mathrm{N})$ sleep and rapid-eye-movements (R) sleep stages have been involved in sleep-dependent memory consolidation processes ${ }^{1,2}$. Evidence points towards a reactivation of neuronal activity during $\mathrm{N}$ sleep through coupled ripples, spindles and slow waves ${ }^{3-6}$, and changes in synaptic plasticity that associate downscaling and upscaling processes through both $N^{7-10}$ and $R^{11-13}$ sleep stages. Yet, beyond the evidence for distinct memory consolidation roles for each sleep stage, it remains unclear whether the succession of the sleep stages within a sleep cycle, with $\mathrm{N}$ systematically preceding $\mathrm{R}$ in physiological sleep, is critical for effective memory consolidation.

Although such a crucial role for the orderly succession of $\mathrm{N}$ then $\mathrm{R}$ sleep stages was previously hypothesized ${ }^{14-18}$, supporting evidence remains scarce. The assumption is mostly supported by the natural sequence of sleep stages and data suggesting a superior effect of the N-R sequence as compared to an isolated $\mathrm{N}$ sleep stage on learning and markers of synaptic plasticity, both in animals ${ }^{14,16,19}$ and humans ${ }^{20-25}$. In humans, support mostly came from perceptual learning paradigms using a visual texture discrimination task $(\mathrm{TDT})^{26}$, which shows a robust sleep-dependent effect on learning ${ }^{27,28}$. It was shown that overnight learning gains best correlate with the combined proportion of $\mathrm{N}$ sleep (especially slow wave sleep, SWS) during early night and R sleep during late night ${ }^{22}$, that a whole night of sleep was more beneficial than only early sleep ${ }^{21}$, or that having both $\mathrm{N}$ (SWS) and R sleep in a sleep nap episode was better than having SWS only ${ }^{23}$. If those last studies may provide indirect support for the sequential hypothesis, they do not control for important factors such as the time spent asleep and in the different sleep stages or for sleep micro-architecture, and they do not assess the importance of the sequence order. The assumptions of the sequential hypothesis are indeed hard to test directly, due to difficulties to evaluate one sleep stage to the exclusion of the other, or to manipulate their order of apparition, especially in humans. Selective deprivation of one stage or the other ${ }^{26}$, sleep restriction in the beginning or the end of the night ${ }^{21}$, 
or morning naps protocols (but see ${ }^{29}$ ) do not enable either to purely isolate one or the other stage or to properly manipulate the order of the sleep stages sequences.

We propose here to bypass those difficulties by testing patients with central hypersomnolence disorder, including patients with narcolepsy who present the unique peculiarity of frequently falling asleep in R sleep before entering $\mathrm{N}$ sleep, although not systematically. We trained them on a visual perceptual discrimination task before and after daytime naps stopped after one sleep cycle either spent in the physiological N-R order, or the anti-physiological R-N order. Our aim was then to analyze the consolidation of perceptual learning across naps according to the N-R and R$\mathrm{N}$ sequence groups and their sleep patterns.

\section{Methods}

\section{Participants}

We systematically proposed the study to inpatients aged 18-45 years diagnosed with narcolepsy type 1 (NT1), type 2 (NT2), or idiopathic hypersomnia (IH), according to International Classification of Sleep Disorders ${ }^{30}$ criteria. All patients had a mean sleep latency to the multiple sleep latency tests $\left(M S L T^{31}\right.$ ) $<8$ min, with at least 2 sleep onsets in $\mathrm{R}$ sleep for patients diagnosed with NT1 and NT2. Patients with NT1 also presented clear cataplexies and were all HLADQB1*0602 positive. HLADQB1*0602 was positive in $38 \%$ of patients with NT2 and $30 \%$ of patients with IH. Participants were recruited at the Sleep and Vigilance department of the Hotel Dieu hospital in Paris (French reference center for central hypersomnia), on a voluntary basis and after providing a written informed consent (Ethical Committee 2016-Mars-14180ND). Exclusion criteria were acute or chronic neurological disorder (other than central hypersomnia), psychiatric disorder, moderate to severe sleep respiratory disorder (apnea-hypopnea index $>15 / \mathrm{h}$ ), or to take medications that could modify the sleep architecture (anti-depressants, hypnotics...). They were requested to stop their psycho- 
stimulant treatments before the day of the experiment for a time of 5 half-lives (i.e., about 75 hours for Modafinil, 60 hours for Pitolisant and 10 hours for methylphenidate). Patients treated with sodium-oxybate were asked not to take the medication the night before the experiment. In total, 41 patients (17 with NT1, 15 with NT2, and 9 with $\mathrm{IH}$ ) met inclusion criteria and accepted to participate in the study (out of 94 queried, 19 did not answer solicitation, 34 did not accept participation, see Figure S1).

\section{Procedure}

Patients proceeded to a general medical evaluation during the inclusion visit 4 to 7 days before the day of experiment, and were asked to fill in questionnaires about their vigilance and sleep quality (Epworth ${ }^{32}$, Karolinska ${ }^{33}$ and Fatigue severity scales ${ }^{34}$, Pittsburgh Sleep Quality Index ${ }^{35}$, morningness-eveningness Horne-Ostberg questionnaire ${ }^{36}$ ), as well as for depression (Beck Depression Inventory $\left.{ }^{37}\right)$. They were also tested for verbal long-term memory $\left(\mathrm{RI}-48{ }^{38}\right.$ ) and vocabulary (Mill Hill scale ${ }^{39}$ ). Besides stopping medications that could interact with sleep and vigilance (see above), they were asked not to drink any stimulant beverage or alcohol from the evening before the experiment and to follow a regular sleep-wake rhythm from the inclusion visit until the experiment (controlled by actimetry - MotionWatch ${ }^{\circledR} 8$, Camntech - delivered at inclusion).

The day of the experiment, patients were tested for visual perceptual learning before and after a nap scheduled two to three times during the same day (i.e., around 9:00, 12:00 am and 3:00 pm). Nineteen out of the 39 patients only slept for two naps because they spent too much time being tested and/or asleep. The average duration of the learning paradigm was about 30 minutes (depending on participant's performance, cf. below). Participants performed a PVT (Psychomotor Vigilance Task ${ }^{40}$ ) and a KSS (Karolinska scale) before the pre-nap task, Visual Analog Scales (VAS) for sleepiness, fatigue and effort after the pre-nap task, and again a KSS before the post-nap task (see Table 1). Naps (lights off) were started within the 15-20 min after the pre-nap task, and after the calibration of polysomnographic recordings. Once the participants woke up from the nap, they could 
walk outside from the room to refresh and release potential sleep inertia (as evidenced by sleepiness assessments before starting the post-nap task). The post-nap task was started after around $30 \mathrm{~min}$ post waking. Patients' naps were categorized depending on the sequence of sleep states within the nap (N-R or R-N sleep).

\section{Learning paradigm: the visual Texture Discrimination Task (vTDT)}

Participants had to discriminate patterns on a visual texture (target) whose visibility was altered by the presentation of a visual mask after a short, controlled time interval (Figure 1A), i.e. the stimulus onset asynchrony (SOA) between the target and the mask (ranging 600 to $40 \mathrm{msec}$, see below). The visual texture was made of $19 \times 19$ horizontal white bars on a black screen, within which were simultaneously presented (1) centrally (foveal vision) a rotated $T$ or $L$ and (2) laterally (peripheral vision) in one visual quadrant a pattern of 3 tilted lines constituting either a horizontal or vertical orientation. Stimuli were presented in Matlab R.2014.b ${ }^{41}$ using the Psychtoolbox extensions 42-44. Participants had to fixate the center of the screen all along the trial and subsequently report using the keyboard 1) what letter was presented and 2) what was the orientation of the 3 oblique bars. The letter detection task aimed at obliging participants to keep the eyes fixated on the central point while processing the orientation in the peripheral visual quadrant. Each block was constituted by 50 trials with the same SOA. The experiment started with a SOA of $600 \mathrm{~ms}$ and on the 3 first trials the target was presented during 5 seconds to ensure a good understanding of the task requirements. The following blocks were then presented with a progressively shortened SOA of 460, $360,260,240,200,160,140,120,100,80,60$, and $40 \mathrm{~ms}$. The task was stopped as soon as accuracy in a block fell below 55\% accuracy (hazard level), computed (in Matlab after each block) as the number of trials with good responses to the orientation on the total number of trials with good letter responses. Blocks in which accuracy for letter detection was below $80 \%$ were discarded from the analysis, as they reflected a poor central fixation. Letters and orientations were pseudorandomly presented within blocks. The oblique bars were presented in a predetermined peripheral quadrant for a given nap session (pre and post nap tests), since improvement in this task has been 
shown to be visual quadrant-dependent ${ }^{45,46}$. One different quadrant was randomly assigned for each nap session.

Behavioral performance was assessed by the threshold-SOA for discrimination, defined as the shorter SOA in which both letter and orientation accuracy was $\geq 80 \%$. It was calculated using a curve fitting relating orientation accuracies and SOA values in the different blocks to limit the effects of performance fluctuations in patients ${ }^{24}$. Curve fitting was obtained using the model $y=a x^{b}+c$ in the Matlab Curve Fitting Toolbox. Perceptual consolidation over nap was assessed comparing thresholdSOAs in post and pre nap tests $(\triangle \mathrm{SOA})$.

\section{Sleep recording and scoring}

During each nap, sleep was recorded using 12 EEG (Fp1, Fp2, F3, F4, C3, C4, P3, P4, O1, O2, T5, T6) and 2 EOG channels referenced to the contralateral mastoid, and bipolar chin EMG and EKG derivations (Embla A10 system, Somnologica ${ }^{\mathrm{TM}} 2$ software, Medcare; Reykjavik, Iceland). Signals were sampled at $200 \mathrm{~Hz}$ and filtered for notch frequency $(50 \mathrm{~Hz})$. Sleep recordings were monitored online to be able to wake up the patient at the end of the first sleep cycle (N-R or R-N sequence) without letting him enter the following cycle. Data were subsequently scored off-line by a sleep expert (MS) using $30 \mathrm{sec}$. epochs (Noxturnal software v5.1) following the international recommendations for sleep scoring ${ }^{47}$. Naps were categorized a posteriori depending on the sleep sequence ( $N-R, R-N$ sequences, see Figure $1 B$, or $N$ and $R$ sleep if the patient woke up before completing a cycle). Only two patients slept with different sleep sequences during their naps.

Sleep parameters and spectral measures were analyzed using the Fieldtrip toolbox ${ }^{48}$ in Matlab (R2014b). The following variables were analyzed: Total Sleep Time (TST), Time spent in N1 (TN1), N2 (TN2), N3 (TN3), N (TN) and R sleep (TR), spindle density, spindle duration and slow wave activity (SWA) in slow wave sleep, rapid-eye-movement (REM) density and theta activity in R sleep. Sleep spindles in $\mathrm{N}$ sleep and REMs in R sleep were automatically detected by means of thresholding the z-transformed value of the bandpass filtered raw data (the threshold was set to 2 for spindles 
and 5 for REMs). Spindles were detected in N2 and N3 sleep on frontal, central and parietal channels, using a bandpass filter between 11 and $16 \mathrm{~Hz}$, for a duration of 0.5 to 2 sec. Spindle density was computed as the total number of spindles divided by the duration of N2 and N3 sleep $\left(\min ^{-1}\right)$. Spindle duration was computed as the mean duration of spindles (sec.). Rapid eye movements (REMs) were detected in R sleep on EOG channels using a bandpass filter between 1 and $15 \mathrm{~Hz}$, for a duration of 0.1 to $1.5 \mathrm{sec}$. REM density was computed as the total number of REMs divided by the duration of $\mathrm{R}$ sleep $\left(\mathrm{min}^{-1}\right)$. All events were then visually checked, and manually corrected if needed. Spectral power analyses for slow wave activity were performed with a Hanning multitaper filter between $0.5-4 \mathrm{~Hz}(0.5 \mathrm{~Hz}$ steps) on frontal and fronto-polar channels and for theta activity in $\mathrm{R}$ sleep between $4-8 \mathrm{~Hz}(0.5 \mathrm{~Hz}$ steps) on central channels.

\section{Statistical analyses}

Our main aim was to compare consolidation across naps $\left(\triangle \mathrm{SOA}=\right.$ threshold_SOA $\mathrm{A}_{\text {post }}-$ threshold_SOA ${ }_{\text {pre}}$ ) in the $\mathrm{N}-\mathrm{R}$ and $\mathrm{R}-\mathrm{N}$ sequence groups, adjusted for their clinical and sleep parameters. The statistical significance was set at 0.05 . The analyses were performed with Stata/MP 14. $1^{49}$. Description and comparison of clinical and sleep characteristics between the two sequence groups are shown in Table 1. Clinical diagnosis was compared between groups with the Fisher's exact test. The quantitative variables were compared using a mixed linear model with the sequence group as a fixed factor and the nap number and subjects as random factors.

We first used repeated measures ANOVA to analyze SOA according to session (pre- and postnap) and sequence group. To further compare consolidation performance between sequence groups in function of clinical and sleep parameters, we analyzed $\triangle \mathrm{SOA}$ according to the $\mathrm{N}-\mathrm{R}$ and $\mathrm{R}-\mathrm{N}$ sequence group and the following variables: age, diagnosis, TST, TN1, TN2, TN3, TN, TR, spindle density, spindle duration, SWA, REM density, and theta activity in R sleep. We first tested the main effects and interaction term (sequence group + covariate + sequence group*covariate) for each variable in a univariable mixed linear model with the sequence group as a fixed factor and the nap 
number and subjects as random factors. Next, we performed multivariable mixed models (multiple independent variables) of $\triangle \mathrm{SOA}$, still with naps and subjects as random variables, using a stepwise procedure on the basis of the Bayesian and Akaike Information Criteria (BIC and AIC) for the selection of explanatory variables. Only variables that showed a trend to be associated with $\triangle \mathrm{SOA}$ $(p<0.05$ uncorrected) in univariable models were selected for the multivariable models. We verified that the standardized residuals of our final model were normally distributed with graphic representations and we used Breusch-Pagan test to verify the homogeneity of variances. We verified non-collinearity between predictors with the variance inflation factor $(<2.5)$. Post-hoc analyses of correlations of the selected variables with $\triangle \mathrm{SOA}$ within each sequence group were performed with Spearman tests, and comparison between correlation scores ( $\mathrm{rho}_{\mathrm{s}}$ ) with Fisher's Z transformation.

\section{Results}

\section{Clinical and sleep data}

The 41 included patients slept for a total of 102 naps (Figure S1, flow chart). Naps presenting mixed sequences of sleep stages (e.g. N-R-N or R-N-R sequences, $n=6$ ) or isolated $N(n=18)$ or $R$ sleep $(n=2)$ were discarded from the analyses. Nap sessions in which participants did not succeed to achieve an accuracy $>80 \%$ in the first block (600 ms SOA) in the pre- or post-nap test were also discarded ( $n=7)$. Two more nap sessions were rejected due to technical issues. In total, 5 patients were excluded due to the exclusion of all their naps (patients performed 2 to 3 naps during the day). Only 2 participants (NT2 patients) contributed to both the N-R and R-N groups, that is, they slept one nap with a $\mathrm{N}-\mathrm{R}$ sequence and another one with a $\mathrm{R}-\mathrm{N}$ sequence. All other patients presented the same sleep sequence type during their naps. Analysis were conducted on a total of 36 participants (15 NT1, $14 \mathrm{NT2}, 7 \mathrm{IH}$ ), with 18 patients (27 naps) in the N-R group and 20 patients (40 naps) in the $\mathrm{R}-\mathrm{N}$ group. 
The clinical and sleep parameters for $\mathrm{N}-\mathrm{R}$ and $\mathrm{R}-\mathrm{N}$ sequence groups are summarized in Table 1. In both sequence groups, patients exhibited on average an excessive daytime sleepiness (Epworth scale $>10$ ) and a tendency for an altered usual night sleep quality (PSQI $\geq 5$, range for N-R group [210], R-N group [3-11]). They presented normal scores to psychometric evaluations. There was a predominance of NT1 patients (and no IH patients) in the R-N group. Patients in the N-R group showed a slightly advanced chronotype (neutral chronotype range from 42 to 58 ) and slept longer than those in the R-N group, with more N1, N2, and R sleep. There was no difference in the time spent in N3. Spindles (density and duration), slow wave activity and rapid eye movement density were comparable between sequence groups, although theta activity in R sleep was stronger in the R$\mathrm{N}$ sequence group.

\section{Behavioral performance and sleep micro-architecture.}

Performance was assessed by the threshold-SOA, computed as the fitted SOA (root-meansquare error for fitting curve: mean $=0.063, S D=0.016$ ) to which subject performed at $80 \%$ of accuracy. Sleep-dependent consolidation of perceptual learning was assessed as the difference between pre- and post-nap threshold SOAs $\left(\triangle \mathrm{SOA}=\right.$ threshold-SOA $\mathrm{Aost}_{\text {- }}$ - threshold-SOA $\left.\mathrm{Are}\right)$, meaning negative values reflect over-nap performance improvement since threshold-SOA is shorter in the post versus the pre-nap condition.

Performance remained stable after vs. before the nap, and did not differ between the 2 sequence groups (threshold-SOA in pre- and post-nap sessions in the N-R group: $146.9-154.4 \mathrm{~ms}$; in the R-N group: 164.0 - $167.3 \mathrm{~ms}$; ANOVA(1,75): NS for main effects of sequence group, session, and interaction sequence group $X$ session, $p=0.16,0.61$, and 0.84 respectively). However, confounding factors such as sleep architecture need to be considered when comparing behavioural performance. Markers of sleep architecture and micro-architecture are precisely the potential factors influencing 
memory consolidation. We then compared performances between sequence groups depending on the different sleep variables.

We first performed univariable mixed linear models to select the variables associated to consolidation. Those models showed a trend for REM density to be negatively associated with consolidation $(\triangle \mathrm{SOA}[\mathrm{IC} 95 \%]=0.003[3.28 \mathrm{e}-4 ; 0.005], \mathrm{p}=0.028$ uncorrected, Table S1), and for a differential effect of spindle duration in function of the sequence group (interaction spindle duration*sequence group, $\triangle \mathrm{SOA}[\mathrm{IC} 95 \%]=0.23[0.076 ; 0.50], \mathrm{p}=0.010$ uncorrected, Table S1). We then performed multivariable linear regressions using a step by step procedure, adding progressively the sequence group, spindle duration, the interaction spindle duration*sequence group and REM density as covariates (see Tables $\mathrm{S} 2$ to $\mathrm{S} 5$ for statistical details of each model). The best multivariable model resulting from the stepwise procedure was the last model including all those covariates $\left(R^{2}=\right.$ 0.406, $F(4,62)=3.05, p=0.023, \mathrm{AIC}=-241, \mathrm{BIC}=-228$, Tables 2 and S5). It confirmed the significant interaction between spindle duration and sequence group ( $p=0.023$, Table $S 5)$, with a main effect of spindle duration in favor of a better over-nap consolidation $(p=0.048)$, and on the contrary the negative association of REM density in R sleep with memory consolidation ( $p=0.028)$.

Post-hoc analyses revealed that consolidation performance increased with the duration of sleep spindles in the N-R group but not in the R-N group, in whom on the contrary analyses showed a trend for a negative association with consolidation (Figure 2, coefficients of correlation of spindles duration with performance in the N-R group: hho $_{s}=-0.53, p=0.027, \mathrm{R}-\mathrm{N}$ : rho $\mathrm{s}_{s}=0.43, \mathrm{p}=0.059$; Fisher test between the 2 rho $\left._{s}: z=-2.96, p=0.0016\right)$. Spindle density were not associated with consolidation in the multivariable model, but to further explore the effect of the different characteristics of sleep spindles, we performed the same analyses on spindle density. There was a similar trend for a better consolidation in the N-R sequence group than in the R-N group with the increase of spindle density (Fisher test: $z=-1.76, p=0.039$, Figure 2 ). Hence, the effect of spindles on memory consolidation appears to be conditioned by the order of sleep stages, i.e. sleep spindles would be associated with 
memory consolidation only in the context of the physiological N-R sleep sequence, providing support to the sequential hypothesis ${ }^{14}$. In post-hoc analyses, the negative association of REM density with consolidation was significant only in the $\mathrm{N}-\mathrm{R}$ group $\left(\mathrm{rho}_{\mathrm{s}}=0.51, \mathrm{p}=0.032\right.$, Figure 2$)$. Other sleep parameters such as the time spent in the different sleep stages, or slow wave and theta activities, were not associated with consolidation (Figure S2).

\section{Discussion}

To assess the sequential hypothesis for memory consolidation ${ }^{14,50}$ that postulates a distinct and complementary role of successive $\mathrm{N}$ and $\mathrm{R}$ stages in a sleep cycle in humans, we tested patients with central hypersomnolence disorders on a visual perceptual learning task before and after naps within which the order of sleep stages could vary. The evolution of performance from pre- to postnap performances, reflecting effective consolidation, was compared between naps featuring the physiological N-R versus the reversed R-N sleep sequence. Our results show that sleep spindles in N sleep, a well-known marker of memory consolidation during sleep, are associated with increased performance after naps only when they are followed by $\mathrm{R}$ sleep, and not when $\mathrm{R}$ precedes $\mathrm{N}$ sleep. Those results provide, to the best of our knowledge, the first direct experimental support to the sequential hypothesis of sleep for memory consolidation in humans.

The strength of this study is to be able to discriminate between a simple dual and independent process of $\mathrm{N}$ and $\mathrm{R}$ sleep on memory consolidation ${ }^{51}$, and a sequential process, in which sleep stages are not only interdependent but also their succession order is important ${ }^{14,18,19}$. Here, we directly compared the effect of post-training N-R and R-N sleep sequences on memory consolidation for perceptual learning. Until now, such a sequential hypothesis was only indirectly suggested through results based on early versus late night protocols, featuring respectively more $\mathrm{N}$ or R sleep ${ }^{21,22}$, after a night with disturbed versus preserved sleep cycles ${ }^{20}$, or on naps featuring $\mathrm{N}$ 
sleep versus $\mathrm{N}-\mathrm{R}$ sleep ${ }^{23}$. However, factors such as the time spent in the different sleep stages and the micro-architecture of sleep were not taken into account so far. In night protocols, the succession of the sleep cycles prevents to control for one specific sequence compared to the other, and in nap protocols, contrasting the $\mathrm{N}-\mathrm{R}$ versus isolated $\mathrm{N}$ conditions had the strong limit to compare a truncated versus the complete sequence, with thus different sleep stages and time spent asleep and no possibility to assess the role of the ordered sequence. In our study, testing patients with central hypersomnolence disorders, and especially narcolepsy, enabled to bypass those limitations. Narcolepsy is a very rare disease (200-500 per million individuals in Europe and North America) characterized by an excessive daytime sleepiness and disturbed sleep, with sleep episodes abnormally starting in $\mathrm{R}$ sleep. Studying this population, although it is difficult to reach a large sample size due to its rare prevalence, is then a unique opportunity to directly test the sequential hypothesis. Here, the sleeping episodes were rigorously stopped after one single N-R or R-N cycle, any occurrence of sleep spindles after the N-R sequence or of REMs after the R-N sequence leading to the rejection of the particular nap session. We also analyzed differences in sleep time and architecture.

Behavioral analyses showed that performances were stable after post-training naps in both sequence types ( $\mathrm{N}-\mathrm{R}$ or $\mathrm{R}-\mathrm{N}$ sleep), suggesting that naps prevented performance deterioration over time, according to previous literature showing a beneficial effect of sleep in healthy participants to counteract experience-dependent deterioration of performance over successive episodes at wake ${ }^{22,28}$. Of note, naps were also shown to increase performance on this task, especially when it was long enough to feature both $\mathrm{N}$ and $\mathrm{R}$ within the sleep episode ${ }^{23}$. The fact that we found here a stabilization rather than an enhancement effect after naps composed of both $\mathrm{N}$ and $\mathrm{R}$ sleep might be linked to the too short duration of naps, or to the pathological status of our patients that might limit their learning capacity, patients with narcolepsy presenting general lower performances in pre- and post-nap tests for visual perceptual learning ${ }^{24}$. Nonetheless, when taking into account the contribution of the phasic components of $\mathrm{N}$ (i.e., spindles) and $\mathrm{R}$ (i.e., REMs) sleep on over-nap 
performance changes, we highlighted the effect of sleep on the consolidation of previous learning, and especially the role of the sleep sequence.

Critically, we found a different and opposite association between spindle duration and performance depending on the sleep sequence, with a positive association only in the N-R condition and not in the R-N one. The association between sleep spindles, neuronal plasticity and memory consolidation is now well established ${ }^{52}$. Especially in visual perceptual learning tasks, the link between sigma activity, the frequency band associated with sleep spindles, and memory consolidation has also already been highlighted ${ }^{53,54}$. However, the interaction with the sleep sequence had not been explored, $\mathrm{R}$ sleep systematically following $\mathrm{N}$ sleep in physiological conditions. Our results show that $\mathrm{R}$ following $\mathrm{N}$ sleep is needed for the sleep spindles to be efficient in memory consolidation. On the contrary, we observed a trend for a deleterious effect of sleep spindles on consolidation when occurring after $\mathrm{R}$ sleep in the $\mathrm{R}-\mathrm{N}$ sequences. Such a negative correlation with performance and markers of synaptic plasticity was already found in rats when $\mathrm{N}$ sleep was followed by wakefulness and not R sleep ${ }^{55}$. Blanco et al. ${ }^{19}$ also recently showed in rats that the N-R sleep transition was critical for synaptic restructuring, with a significant increase in synaptic weight associated with cortical spindles near the N-R transitions. Altogether, those results suggest that N/SWS sleep and associated markers would lead to synaptic downscaling without restructuring and proper consolidation of newly acquired memories when not followed by $\mathrm{R}$ sleep.

No association was found with slow wave activity in $\mathrm{N}$ sleep, an effect inconstantly found in the literature of perceptual learning ${ }^{53,56}$, or with theta activity in R sleep. However, unexpectedly, we found a deleterious effect of REMs in R sleep on memory consolidation, independently of the sleep sequence. While $R$ sleep has been positively involved in perceptual learning ${ }^{23,45,57,58}$, no study analyzed so far the role of REMs. Yet, recent literature suggests that phasic and tonic R states likely cover distinctive functions ${ }^{59}$, and that $\mathrm{R}$ sleep may play an important role in the active forgetting of memories ${ }^{60}$. Another explanation could be that REMs and associated activity in visual cortical areas 
may interact with mechanisms of synaptic plasticity during sleep and prevent the good consolidation of visual perceptual learning. Further studies are needed to precise this effect, but this result highlights the intricate role of sleep stages in promoting or not memory consolidation.

Our study presents several limitations, mainly driven by the inherent characteristics of the patient population. First, central hypersomnolence disorders are rare and the number of patients in each sequence group was limited. Second, we included patients with different type of central hypersomnolence disorders and not only patients with NT1, who are the patients with the most homogeneous phenotype, because they fall more frequently asleep in $R$ sleep, leading to unbalanced sequence groups (in our sample only 2 out of the 15 patients with NT1 slept in the N-R sequence). We included patients with both NT2 and HI because of the recognized overlap in those diseases ${ }^{61-63}$. Then, the characteristic of naps in the physiological $N-R$ sequences differed from the $\mathrm{R}-\mathrm{N}$ naps with especially a longer time spent in N2 sleep, probably reflecting an inherent characteristic of the R-N sequence. However, knowing those differences in clinical and sleep parameters between the sequence groups, we tested their association with consolidation performances in our statistical models. Diagnosis type was not associated with consolidation, neither as the time spent in the different sleep stages. Also, sleepiness assessments before and after naps showed that there was no difference between sequence groups, suggesting that potential sleep inertia especially seen in patients with IH could have been correctly released before testing.

As we tested patients with central hypersomnia and not healthy subjects, we may question the generalization of our results to the general population. However, no alteration of $\mathrm{N}$ sleep, and especially sleep spindles, was shown in patients with NT1 or NT2 ${ }^{64}$. Also, the effect of sleep spindles was reinforced when pooling all the patients with central hypersomnia, suggesting that it was not specific to one population or disease type. Considering patients with narcolepsy, the inversion of the $\mathrm{N}-\mathrm{R}$ sleep sequence may be an important factor interacting with consolidation mechanisms. If memory performance in usual tests seems to be mainly preserved in narcoleptic patient ${ }^{65-67}$, 
hippocampal volume in narcoleptic patients has been shown to be decreased compared to control subjects, and this decreased volume was correlated with shorter $\mathrm{R}$ sleep latencies ${ }^{67}$. Moreover, patients with narcolepsy showed poorer performance when presenting more sleep onset in R sleep in the same TDT task ${ }^{24}$. We demonstrate here that sleep onset in $\mathrm{R}$ sleep has a direct negative impact on memory consolidation processes. The succession of sleep cycles along the night may, however, help to prevent strong memory dysfunction in those patients.

In conclusion, we show that markers of $\mathrm{N}$ sleep involved in memory consolidation such as sleep spindles are beneficial only when followed by R sleep, supporting the sequential hypothesis for memory consolidation in humans. The negative effect of REMs in R sleep on consolidation highlights the subtle and balanced role of sleep stages both to remember and to forget. Finally, our results provide insight on how the reversion of sleep stages in narcolepsy may impair learning and memory processes in this population. 


\section{Acknowledgements}

We warmly thank all the patients who participated in the study. We thank M. DramaixWilmet for her help with the statistical aspects.

M.S. made contributions to the conception and design of the work, to data acquisition, analysis, interpretation, and have drafted the manuscript, L.G., P.V.B., M.E, M.C. made contributions to data acquisition, J.B. and F.S. made contributions to statistical analyses and reporting, D.L. and P.P. made contributions to the conception of the work, data interpretation and have revised the manuscript.

\section{Disclosure Statement}

(a) Financial disclosure statement: Damien Léger declares that in the past 5 years he has been employed as an investigator or a consultant by Actellion-Idorsia, the Agence Spatiale Européenne, Bioprojet, iSommeil, ESAI, Jansenn, Jazz, Vanda, Merck, Philips, Rythm, Sanofi, Vitalaire, and Resmed.

(b) Non-financial disclosure statement: none.

\section{Data Availability Statements}

The data underlying this article will be shared on reasonable request to the corresponding author. 


\section{References}

1. Peigneux P, Laureys S, Delbeuck X, Maquet P. Sleeping brain, learning brain. The role of sleep for memory systems. Neuroreport. 2001;12(18):A111-124.

2. Rasch B, Born J. About Sleep's Role in Memory. Physiol Rev. 2013;93(2):681-766. doi:10.1152/physrev.00032.2012

3. Steriade $\mathrm{M}$, Contreras $\mathrm{D}$, Dossi RC, Nunez A. The slow (\&lt; $1 \mathrm{~Hz}$ ) oscillation in reticular thalamic and thalamocortical neurons: scenario of sleep rhythm generation in interacting thalamic and neocortical networks. J Neurosci. 1993;13(8):3284-3299. doi:10.1523/JNEUROSCI.13-0803284.1993

4. Mölle M, Marshall L, Gais S, Born J. Grouping of Spindle Activity during Slow Oscillations in Human Non-Rapid Eye Movement Sleep. J Neurosci. 2002;22(24):10941-10947.

5. Steriade M. Grouping of brain rhythms in corticothalamic systems. Neuroscience. 2006;137(4):1087-1106. doi:10.1016/j.neuroscience.2005.10.029

6. $\quad$ Clemens Z, Mölle M, Erőss L, Barsi P, Halász P, Born J. Temporal coupling of parahippocampal ripples, sleep spindles and slow oscillations in humans. Brain. 2007;130(11):28682878. doi:10.1093/brain/awm146

7. Tononi G, Cirelli C. Sleep and synaptic homeostasis: a hypothesis. Brain Res Bull. 2003;62(2):143-150. doi:10.1016/j.brainresbull.2003.09.004

8. Tononi G, Cirelli C. Sleep and synaptic down-selection. Eur J Neurosci. 2020;51(1):413-421. doi:10.1111/ejn.14335

9. Chauvette S, Seigneur J, Timofeev I. Sleep Oscillations in the Thalamocortical System Induce Long-Term Neuronal Plasticity. Neuron. 2012;75(6):1105-1113. doi:10.1016/j.neuron.2012.08.034

10. Timofeev I, Chauvette S. Sleep slow oscillation and plasticity. Curr Opin Neurobiol. 2017;44:116-126. doi:10.1016/j.conb.2017.03.019

11. Ribeiro S, Goyal V, Mello CV, Pavlides C. Brain Gene Expression During REM Sleep Depends on Prior Waking Experience. Learn Mem. 1999;6(5):500-508.

12. Ribeiro S, Mello CV, Velho T, Gardner TJ, Jarvis ED, Pavlides C. Induction of Hippocampal Long-Term Potentiation during Waking Leads to Increased Extrahippocampal zif-268 Expression during Ensuing Rapid-Eye-Movement Sleep. J Neurosci. 2002;22(24):10914-10923. doi:10.1523/JNEUROSCI.22-24-10914.2002

13. Grosmark AD, Mizuseki K, Pastalkova E, Diba K, Buzsáki G. REM Sleep Reorganizes Hippocampal Excitability. Neuron. 2012;75(6):1001-1007. doi:10.1016/j.neuron.2012.08.015

14. Giuditta A, Ambrosini MV, Montagnese $P$, et al. The sequential hypothesis of the function of sleep. Behav Brain Res. 1995;69(1-2):157-166.

15. Diekelmann S, Born J. The memory function of sleep. Nat Rev Neurosci. 2010;11(2):114-126. 
doi:10.1038/nrn2762

16. Ribeiro S. Sleep and plasticity. Pflugers Arch. 2012;463(1):111-120. doi:10.1007/s00424-011$1031-5$

17. Deliens G, Leproult R, Neu D, Peigneux P. Rapid Eye Movement and Non-Rapid Eye Movement Sleep Contributions in Memory Consolidation and Resistance to Retroactive Interference for Verbal Material. SLEEP. Published online December 1, 2013. doi:10.5665/sleep.3220

18. Seibt J, Frank MG. Primed to sleep: The dynamics of synaptic plasticity across brain states. Front Syst Neurosci. 2019;13. doi:10.3389/fnsys.2019.00002

19. Blanco W, Pereira CM, Cota VR, et al. Synaptic Homeostasis and Restructuring across the Sleep-Wake Cycle. PLoS Comput Biol. 2015;11(5):e1004241. doi:10.1371/journal.pcbi.1004241

20. Ficca G, Lombardo $P$, Rossi L, Salzarulo P. Morning recall of verbal material depends on prior sleep organization. Behav Brain Res. 2000;112(1-2):159-163. doi:10.1016/s0166-4328(00)00177-7

21. Gais S, Plihal W, Wagner U, Born J. Early sleep triggers memory for early visual discrimination skills. Nat Neurosci. 2000;3(12):1335-1339. doi:10.1038/81881

22. Stickgold R, Whidbee D, Schirmer B, Patel V, Hobson JA. Visual discrimination task improvement: A multi-step process occurring during sleep. J Cogn Neurosci. 2000;12(2):246-254.

23. Mednick S, Nakayama K, Stickgold R. Sleep-dependent learning: a nap is as good as a night. Nat Neurosci. 2003;6(7):697-698. doi:10.1038/nn1078

24. Cipolli C, Campana G, Campi C, et al. Sleep and time course of consolidation of visual discrimination skills in patients with narcolepsy-cataplexy. J Sleep Res. 2009;18(2):209-220. doi:10.1111/j.1365-2869.2008.00712.x

25. Batterink $L$, Westerberg C, Paller KA. Vocabulary learning benefits from REM after slowwave sleep. Neurobiol Learn Mem. 2017;144:102-113. doi:10.1016/j.nlm.2017.07.001

26. Karni A, Sagi D. The time course of learning a visual skill. Nature. 1993;365(6443):250-252. doi:10.1038/365250a0

27. Stickgold $R$, James $L$, Hobson JA. Visual discrimination learning requires sleep after training. Nat Neurosci. 2000;3(12):1237-1238. doi:10.1038/81756

28. Mednick SC, Nakayama K, Cantero JL, et al. The restorative effect of naps on perceptual deterioration. Nat Neurosci. 2002;5(7):677-681. doi:10.1038/nn864

29. Tinguely G, Huber R, Borbély AA, Achermann P. Non-rapid eye movement sleep with low muscle tone as a marker of rapid eye movement sleep regulation. BMC Neurosci. 2006;7:2. doi:10.1186/1471-2202-7-2

30. ICSD-3. The International Classification of Sleep Disorders. American Academy of Sleep Medicine Darien, IL; 2014. 
31. Carskadon MA, Dement WC. The Multiple Sleep Latency Test: What Does It Measure? Sleep. 1982;5(suppl_2):S67-S72. doi:10.1093/sleep/5.S2.S67

32. Johns MW. A new method for measuring daytime sleepiness: the Epworth sleepiness scale. Sleep. 1991;14(6):540-545.

33. Akerstedt T, Gillberg M. Subjective and objective sleepiness in the active individual. Int J Neurosci. 1990;52(1-2):29-37. doi:10.3109/00207459008994241

34. Krupp LB, LaRocca NG, Muir-Nash J, Steinberg AD. The fatigue severity scale. Application to patients with multiple sclerosis and systemic lupus erythematosus. Arch Neurol. 1989;46(10):11211123. doi:10.1001/archneur.1989.00520460115022

35. Buysse DJ, Reynolds CF 3rd, Monk TH, Berman SR, Kupfer DJ. The Pittsburgh Sleep Quality Index: a new instrument for psychiatric practice and research. Psychiatry Res. 1989;28(2):193-213.

36. Horne JA, Ostberg O. A self-assessment questionnaire to determine morningnesseveningness in human circadian rhythms. Int J Chronobiol. 1976;4(2):97-110.

37. Beck AT, Steer RA, Brown G. Beck depression inventory-II. Psychol Assess. Published online 1996.

38. Adam S. Tâche de Rappel Indicé à 48 items (RI-48). Solal; 2008. Accessed September 18, 2020. https://orbi.uliege.be/handle/2268/69746

39. Colman AM. Mill Hill Vocabulary scale. In: A Dictionary of Psychology. Oxford University Press; 2009. Accessed September 18, 2020.

https://www.oxfordreference.com/view/10.1093/acref/9780199534067.001.0001/acref9780199534067-e-5086

40. Dinges DF, Powell JW. Microcomputer analyses of performance on a portable, simple visual RT task during sustained operations. Behav Res Methods Instrum Comput. 1985;17(6):652-655. doi:10.3758/BF03200977

41. MATLAB. version 14 (R2014b). Natick, Massachusetts: The MathWorks Inc.; 2014.

42. Brainard DH. The Psychophysics Toolbox. Spat Vis. 1997;10(4):433-436. doi:10.1163/156856897X00357

43. Pelli DG. The VideoToolbox software for visual psychophysics: Transforming numbers into movies. Spat Vis. 1997;10(4):437-442. doi:10.1163/156856897X00366

44. Kleiner M, Brainard D, Pelli D, Ingling A, Murray R, Broussard C. What's new in psychtoolbox3. Perception. 2007;36(14):1-16.

45. Karni A, Tanne D, Rubenstein BS, Askenasy JJ, Sagi D. Dependence on REM sleep of overnight improvement of a perceptual skill. Science. 1994;265(5172):679-682.

46. Deliens G, Schmitz R, Peigneux P. Interocular transfer of perceptual skills after sleep. J Vis. 2014;14(1):23-23. doi:10.1167/14.1.23 
47. American Academy of Sleep Medicine, Iber C. The AASM Manual for the Scoring of Sleep and Associated Events: Rules, Terminology and Technical Specifications. American Academy of Sleep Medicine; 2007.

48. Oostenveld R, Fries P, Maris E, Schoffelen JM. FieldTrip: Open Source Software for Advanced Analysis of MEG, EEG, and Invasive Electrophysiological Data. Comput Intell Neurosci. 2011;2011:1-9. doi:10.1155/2011/156869

49. StataCorp. 2015. Stata Statistical Software: Release 14. College Station, TX: StataCorp LP.

50. Ambrosini MV, Sadile AG, Gironi Carnevale UA, Mattiaccio M, Giuditta A. The sequential hypothesis on sleep function. I. Evidence that the structure of sleep depends on the nature of the previous waking experience. Physiol Behav. 1988;43(3):325-337. doi:10.1016/0031-9384(88)90196-5

51. Plihal W, Born J. Effects of Early and Late Nocturnal Sleep on Declarative and Procedural Memory. J Cogn Neurosci. 1997;9(4):534-547. doi:10.1162/jocn.1997.9.4.534

52. Peyrache A, Seibt J. A mechanism for learning with sleep spindles. Philos Trans $R$ Soc $B$ Biol Sci. 2020;375(1799):20190230. doi:10.1098/rstb.2019.0230

53. Bang JW, Khalilzadeh $\mathrm{O}$, Hämäläinen $\mathrm{M}$, Watanabe T, Sasaki Y. Location specific sleep spindle activity in the early visual areas and perceptual learning. Vision Res. 2014;99:162-171.

doi:10.1016/j.visres.2013.12.014

54. Tamaki M, Wang Z, Watanabe T, Sasaki Y. Trained-feature-specific offline learning by sleep in an orientation detection task. J Vis. 2019;19(12):12. doi:10.1167/19.12.12

55. Langella M, Colarieti L, Ambrosini MV, Giuditta A. The sequential hypothesis of sleep function. IV. A correlative analysis of sleep variables in learning and nonlearning rats. Physiol Behav. 1992;51(2):227-238. doi:10.1016/0031-9384(92)90135-0

56. Aeschbach D, Cutler AJ, Ronda JM. A Role for Non-Rapid-Eye-Movement Sleep Homeostasis in Perceptual Learning. J Neurosci. 2008;28(11):2766-2772. doi:10.1523/JNEUROSCI.5548-07.2008

57. Mascetti L, Muto V, Matarazzo L, et al. The impact of visual perceptual learning on sleep and local slow-wave initiation. J Neurosci Off J Soc Neurosci. 2013;33(8):3323-3331.

doi:10.1523/JNEUROSCI.0763-12.2013

58. McDevitt EA, Duggan KA, Mednick SC. REM sleep rescues learning from interference. Neurobiol Learn Mem. 2015;122:51-62. doi:10.1016/j.nIm.2014.11.015

59. Simor P, van der Wijk G, Nobili L, Peigneux P. The microstructure of REM sleep: Why phasic and tonic? Sleep Med Rev. 2020;52:101305. doi:10.1016/j.smrv.2020.101305

60. Izawa S, Chowdhury S, Miyazaki T, et al. REM sleep-active $\mathrm{MCH}$ neurons are involved in forgetting hippocampus-dependent memories. Science. 2019;365(6459):1308-1313.

doi:10.1126/science.aax9238

61. Šonka K, Šusta M, Billiard M. Narcolepsy with and without cataplexy, idiopathic hypersomnia with and without long sleep time: a cluster analysis. Sleep Med. 2015;16(2):225-231. 
doi:10.1016/j.sleep.2014.09.016

62. Lammers GJ, Bassetti CLA, Dolenc-Groselj L, et al. Diagnosis of central disorders of hypersomnolence: A reappraisal by European experts. Sleep Med Rev. 2020;52:101306. doi:10.1016/j.smrv.2020.101306

63. Fronczek R, Arnulf I, Baumann CR, Maski K, Pizza F, Trotti LM. To split or to lump? Classifying the central disorders of hypersomnolence. Sleep. 2020;43(8). doi:10.1093/sleep/zsaa044

64. Christensen JAE, Nikolic M, Hvidtfelt M, Kornum BR, Jennum P. Sleep spindle density in narcolepsy. Sleep Med. 2017;34:40-49. doi:10.1016/j.sleep.2017.02.022

65. Aguirre M, Broughton R, Stuss D. Does memory impairment exist in narcolepsy-cataplexy? J Clin Exp Neuropsychol. 1985;7(1):14-24. doi:10.1080/01688638508401239

66. Rogers AE, Rosenberg RS. Tests of memory in narcoleptics. Sleep. 1990;13(1):42-52.

67. Joo EY, Kim SH, Kim ST, Hong SB. Hippocampal volume and memory in narcoleptics with cataplexy. Sleep Med. 2012;13(4):396-401. doi:10.1016/j.sleep.2011.09.017 


\section{Figure Captions}

Figure 1. Experimental design. (A) Representation of one trial. Participants have to discriminate within a visual texture 1 ) the central letter ( $T$ or $L$ ) and 2 ) the orientation of a pattern of oblique bars (vertical or horizontal) presented in one peripheral quadrant. Perception is made more difficult from one block (50 trials) to the other with the presentation of a subsequent mask at decreasing stimulus onset asynchrony (SOA; from 600 to $40 \mathrm{~ms}$ ). The task is presented before and after a nap. (B) Hypnograms of two representative naps. Nap sessions are categorized based on the sleep sequence during the nap: N-R or R-N sleep sequence.

Figure 2. Phasic events of sleep and performance. Sleep spindles are associated with better consolidation only when $\mathrm{N}$ is followed by $\mathrm{R}$ sleep. Rapid-eye-movements in $\mathrm{R}$ sleep are negatively associated with consolidation, whatever the sleep sequence. Corresponding multivariable model is described in Table 2. Unlike spindle duration and REM density, spindle density was not associated with consolidation in the linear regression model and is shown here to illustrate a trend. Post-hoc correlations of selected variables with consolidation were performed using Spearman tests for each sequence group and Fisher tests were used for the comparison between sequence groups. $\triangle \mathrm{SOA}=$ $\mathrm{SOA}_{\text {post }}-\mathrm{SOA}_{\text {pre }}$ in the N-R (cyan) and R-N (red) sequence groups. 


\section{Tables}

Table 1. Clinical and sleep parameters

\begin{tabular}{|c|c|c|c|}
\hline & $N-R(n=17)$ & $R-N(n=19)$ & $\mathrm{p}$-value ${ }^{\mathrm{a}}$ \\
\hline \multicolumn{4}{|l|}{ Clinical data } \\
\hline Diagnosis & & & $<0.001 * *$ \\
\hline NT1 (n) & 2 & 13 & $\mathrm{~b}$ \\
\hline NT2 (n) & 8 & 6 & \\
\hline $\mathrm{IH}(\mathrm{n})$ & 7 & 0 & \\
\hline Age (years) & $28[26-32]$ & $26[20-35]$ & 0.57 \\
\hline Body Mass Index $\left(\mathrm{kg} / \mathrm{m}^{2}\right)$ & $22.85[20.1-25.27]$ & $23.4[22-30.4]$ & 0.26 \\
\hline \multicolumn{4}{|l|}{ Sleep evaluation } \\
\hline Epworth Sleepiness Scale & $13[8-16]$ & $15[11-18]$ & 0.12 \\
\hline Fatigue Severity Scale & $4.2[3.2-5.6]$ & $3.7[2.9-4.6]$ & 0.32 \\
\hline Pittsburgh Sleep Quality Index & $5[3-6]$ & $6[4-7]$ & 0.43 \\
\hline Horne and Ostberg questionnaire & $40[36-46]$ & $53[46-59]$ & $<0.01 * *$ \\
\hline \multicolumn{4}{|l|}{ Psychometric evaluation } \\
\hline $\mathrm{RI}-48$ & 31.97 [31.73-32.06] & $31.82[30.95-32.21]$ & 0.76 \\
\hline Beck Depression Inventory & $4[1-7]$ & $4[1-6]$ & 0.61 \\
\hline Mill Hill Vocabulary Test & $11[7-16]$ & $11[6-16]$ & 0.79 \\
\hline \multicolumn{4}{|l|}{ Pre-nap, before task } \\
\hline Karolinska Sleepiness Scale & $3[2.5-4]$ & $3[3-4]$ & 0.68 \\
\hline PVT 1/RT & $3.24[3.01-3.43]$ & $3.23[3.04-3.41]$ & 0.79 \\
\hline PVT lapses & $1[0.5-3]$ & $1.5[0.33-3.5]$ & 0.82 \\
\hline \multicolumn{4}{|l|}{ Pre-nap, after task } \\
\hline Sleepiness VAS & $4.5[3.5-5.75]$ & $5[3.67-5]$ & 0.59 \\
\hline Fatigue VAS & $5.75[4-6.5]$ & $5[3.25-6]$ & 0.32 \\
\hline Effort VAS & $5.25[4-7]$ & $5.5[4.83-6]$ & 0.81 \\
\hline \multicolumn{4}{|l|}{ Post-nap, before task } \\
\hline Karolinska Sleepiness Sc & $3[2.5-5]$ & $3[2-4.5]$ & 0.32 \\
\hline \multicolumn{4}{|l|}{ Sleep data } \\
\hline \multicolumn{4}{|l|}{ Time spent in (min) } \\
\hline Total sleep & $77[68.6-82]$ & $51.7[37.1-54.8]$ & $<0.01 *$ \\
\hline N1 & 14 [13.4-18.5] & $5.6[2.3-9.9]$ & $<0.01 * *$ \\
\hline N2 & $29.3[22.5-32.5]$ & $13.5[9.8-15.6]$ & $<0.01 * *$ \\
\hline N3 & $11.3[6.5-18.5]$ & $17.2[6.6-26.1]$ & 0.16 \\
\hline $\mathrm{R}$ & $19.3[12-25]$ & $10.4[8-14.5]$ & $<0.01 * *$ \\
\hline Spindle density $\left(\mathrm{min}^{-1}\right)$ & $7.2[5.7-8]$ & $6.7[4.9-7.6]$ & 0.71 \\
\hline Spindle duration (s) & $0.86[0.82-0.93]$ & $0.83[0.80-0.91]$ & 0.68 \\
\hline SWA $\left(e 04 \mu V^{2}\right)$ & $12[5.3-30.2]$ & $5.5[3.6-8.5]$ & 0.28 \\
\hline REM density $\left(\min ^{-1}\right)$ & $8.5[5.1-11]$ & $6.4[4.4-9.3]$ & 0.41 \\
\hline Theta activity in $\mathrm{R}\left(\mathrm{e} 03 \mu \mathrm{V}^{2}\right)$ & $0.23[0.04-0.79]$ & $1.2[0.74-4.1]$ & $<0.01 * * *$ \\
\hline
\end{tabular}

RI-48: cued recall test (indexed recall / immediate recall). SWA: Slow Wave Activity. REM: rapid eye movement. The values are given as median [p25-p75]. $n=$ number of subjects. 
${ }^{a}$ Mixed multivariable model with nap number and subject as random variables (except for diagnosis ${ }^{b}$ )

${ }^{b}$ Fisher's exact test: NT1/NT2: Bonferroni adjusted $p=0.06 ;$ NT1/IH: adjusted $p<0.001 ; N T 2 / I H$ : adjusted $p=0.18$. 
Table 2. Multivariable mixed linear regression model of consolidation ( $\triangle S O A)$

\begin{tabular}{lllll}
\hline Predictor & Estimate & SE & t & p \\
\hline Sequence group & -0.21486 & 0.09243 & -2.32 & 0.023 \\
REM density & 0.00249 & 0.00110 & 2.25 & 0.028 \\
Spindle duration & -0.39670 & 0.19644 & -2.02 & 0.048 \\
$\begin{array}{l}\text { Spindle duration * Sequence } \\
\text { group }\end{array}$ & 0.24928 & 0.10694 & 2.33 & 0.023 \\
\hline
\end{tabular}

Multivariable model with naps and subjects as random variables, and sequence group, spindle duration, interaction spindle duration*sequence group and REM density as covariates $\left(R^{2}=0.406, F(4,62)=3.05, p=\right.$ $0.023, A I C=-241$ ). 
Figure 1

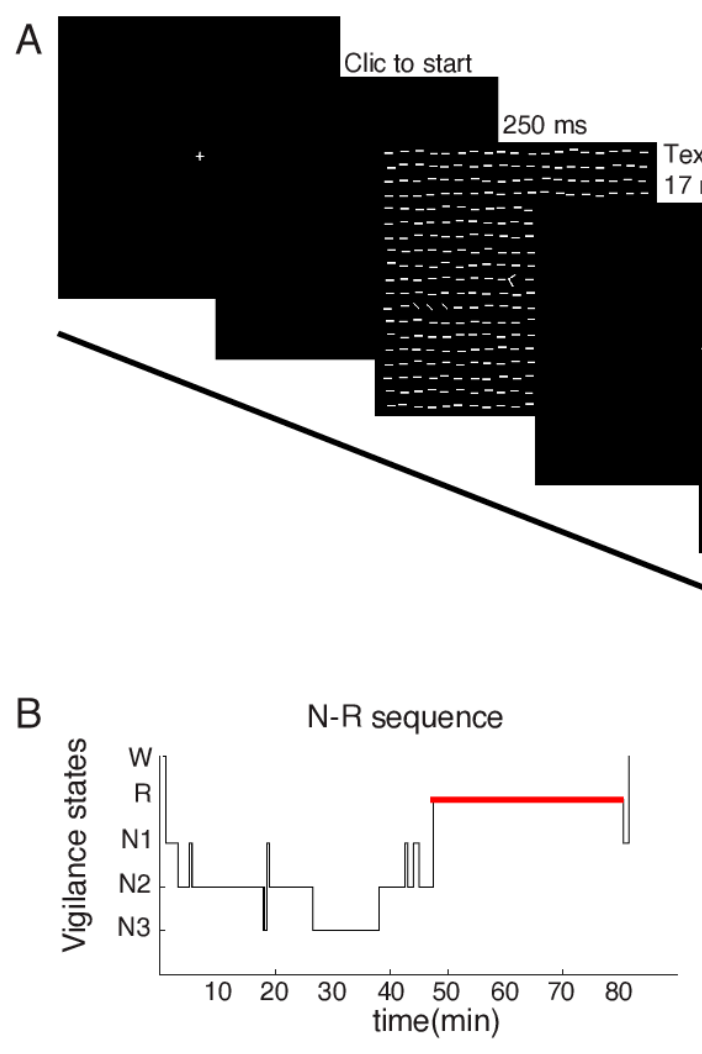

SOA

Variable time

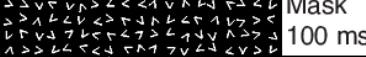

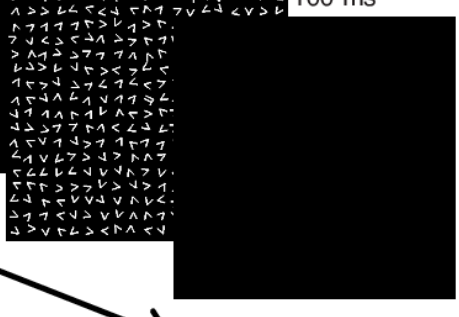

esponse:

1. T or L?

2. Vertical

or horizontal?

B

R-N sequence

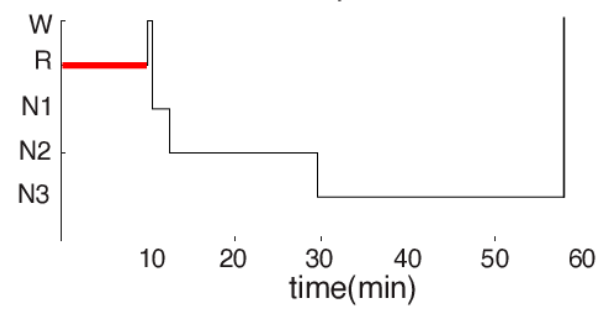


Figure 2
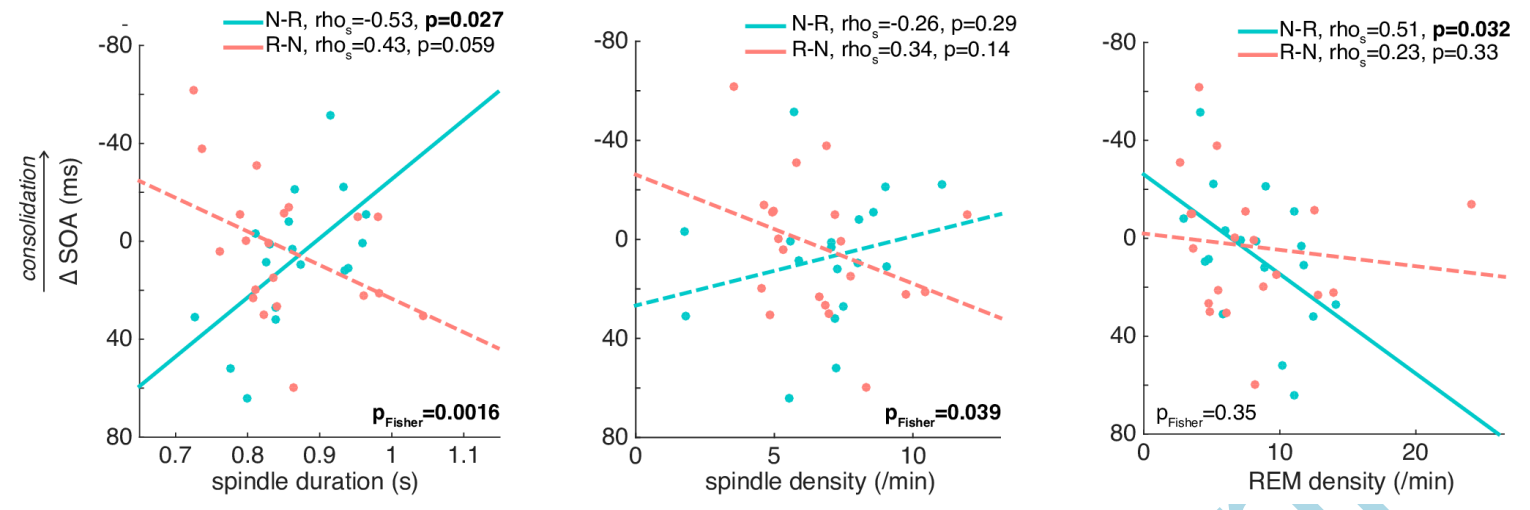